\title{
Acute trauma coagulopathy: prevalence and outcome in trauma patients presenting to the Emergency Department SMBB Trauma Centre, Civil Hospital Karachi.
}

\section{Fariha Ashraf ( $\square$ farihaashraf@gmail.com )}

Dow University of Health Sciences https://orcid.org/0000-0002-0338-755X

Mohammad Faisal Ibrahim

Dow University of Health Sciences

\section{Rabia Feroz}

Dow University of Health Sciences

Abdul Wasey

Karachi Medical and Dental College

Muhammad Arsalan

Dow University of Health Sciences

Mohammad Saeed Quraishy

Dow University of Health Sciences

\section{Research Article}

Keywords: Acute trauma, trauma coagulopathy, Age, Mortality

Posted Date: February 9th, 2021

DOl: https://doi.org/10.21203/rs.3.rs-183028/v1

License: (c) (i) This work is licensed under a Creative Commons Attribution 4.0 International License.

Read Full License 


\section{Abstract}

PURPOSE To determine the prevalence of Acute Trauma Coagulopathy (ATC) in severely injured patients presenting to the emergency department of SMBB Trauma Centre of Civil Hospital Karachi and to investigate its impact on these patient's outcome. Early recognition using initial coagulation profile (PT/APTT) accompanied by adequate management of ATC and prevention of bleeding may substantially reduce mortality and improve outcomes in severely injured patients in resource limited environments.

MATERIAL AND METHODS This descriptive cross sectional study was carried out in Surgical unit 4, Dr Ruth K.M. Pfau Civil hospital Karachi from 27 th July 2019 to 28 th January 2020. After approval from institutional review board, 80 patients aged between 14 to 60 years with injury severity score more than 15 were enrolled using non probability consecutive sampling technique and their coagulation profile (PT, APTT and INR) was done at presentation and followed for in hospital mortality. Data was collected and analyzed using SPSS 24.

RESULTS In our study total 80 patients were enrolled, the mean age of patients was $27.28 \pm 12.18$. Trauma coagulopathy was present in $12.5 \%$ patients. In hospital mortality was found to be $16.3 \%$. The in hospital mortality was compared in patients with coagulopathy and patients without coagulopathy and $p$ value was significant 0.00 . Data stratification was significant for age with $p$ value 0.016 . Data stratification for gender, mode of injury, mechanism of injury, amount of fluid given at presentation and injury severity score was not significant.

CONCLUSION Acute trauma coagulopathy is associated with increased risk of mortality.

\section{Introduction}

Trauma remains a leading cause of death and disability in adults in spite of advances in resuscitation, surgical management, and critical care [1]. The World Health Organization (WHO) estimates that around 5 million people die of injuries every year, accounting for $10 \%$ of global annual mortality [2]. Low- and middle income nations are severely affected with over $90 \%$ of the world's trauma mortality occurring in these nations. Young people are most commonly involved in trauma related deaths with almost $50 \%$ of those who die are between 15 and 44 years of age, with males accounting for twice as many deaths as females; death due to traumatic injury is, therefore, the leading cause of life years lost. Violence-selfinflicted, interpersonal, and war-related-accounts for half of trauma mortality. Road traffic accounts for the next largest proportion [3].

Immediately after trauma a number of physiological derangements set in a patient. Central to trauma management along with determination of the extent of injury is early recognition of these derangements and initiation of goal directed therapy. Hemorrhage is responsible for $40 \%$ of all trauma deaths [4]. A frequent complication of hemorrhage is coagulopathy that may occur in up to $25 \%$ of patients with trauma, even before hospital admission [5]. Acute traumatic coagulopathy (ATC) is an endogenous 
impairment of hemostasis that occurs early after injury and is associated with increased mortality and worse outcomes in the polytrauma patients [6].

Coagulopathy associated with traumatic injury is the result of multiple independent but interacting mechanisms. Early coagulopathy is driven by shock and requires thrombin generation from tissue injury as an initiator. Initiation of coagulation occurs with activation of anticoagulant and fibrinolytic pathways. This Acute Coagulopathy of Trauma-Shock is altered by subsequent events and medical therapies, in particular acidemia, hypothermia, and dilution. There is significant interplay between all mechanisms.

Acute trauma coagulopathy is caused by tissue injury, hypo perfusion and shock. Mechanisms for this acute coagulopathy include activation of protein $\mathrm{C}$, endothelial glycocalyx disruption, depletion of fibrinogen, and platelet dysfunction. Hypothermia and acidaemia amplify the endogenous coagulopathy and often accompany trauma. These multifactorial processes lead to decreased clot strength, autoheparinization, and hyperfibrinolysis. Furthermore, the effects of aggressive crystalloid administration, haemodilution from inappropriate blood product transfusion, and prolonged surgical times may worsen clinical outcomes [7].

Sarah et al. in a retrospective study of combat casualities showed that mortality in those who were coagulopathic at arrival to the emergency department was $24 \%(32 / 133)$ versus $4 \%(8 / 214)$ in those not presenting with coagulopathy [8]. Mujuni et al. in a study carried out in 2012 found out the frequency of patients with acute trauma coagulopathy among major trauma patients to be $54.4 \%$ and a mortality of $29.29 \%$ in patients with ATC as compared to $10.84 \%$ in non ATC patients [2]. Sjoerd et al. in a study of 107 patients reported that early coagulopathy as diagnosed in the emergency department occurred in $24 \%$ of all patients and during the first 24 hours post-trauma it provided an independent prognostic factor for unfavorable outcome (odds ratio (OR) 3.75 (95\% $\mathrm{Cl} 1.07$ to $12.51 ; \mathrm{P}=0.04)$ and $\mathrm{OR} 11.61$ (2.79 to 48.34); $P=0.003)[9]$.

The aim of this study is to determine the prevalence of Acute Trauma Coagulopathy (ATC) in severely injured patients presen 1 ting to the emergency department of Civil Hospital Karachi and to investigate its impact on these patient's outcome. Studies have reported an overall mortality among major trauma patients with acute trauma coagulopathy ranging from $15 \%$ to $24 \%$ [10]. However studies in low and middle income countries like Pakistan are lacking, which are most severely affected and contribute to over $90 \%$ of trauma related mortality. After trauma most preventable deaths occur due to bleeding [11]. Early recognition using initial coagulation profile (PT/APTT) accompanied by adequate and aggressive management of Acute Trauma Coagulopathy and prevention of bleeding may substantially reduce mortality and improve outcomes in severely injured patients in resource limited environments like ours.

\section{Materials And Methods}

STUDY DESIGN: Descriptive Case Series. 
STUDY SETTING: Emergency Department of SMBB Trauma Centre and Surgical Unit 4, DR. RUTH K.M. PFAU Civil Hospital Karachi.

Duration of Study: 27th July 2019 to 28th January 2020.

Sample Size: Using WHO Calculator Statistics for Mortality in patients with ATC - 29.29\% d - 10\% (Sample Size $n-80$ )

Sampling Technique: Non-probability consecutive sampling.

Inclusion Criteria:

1. All patients with injuries to head and neck, face, thorax, abdomen, extremities, external (surface) with Injury Severity Score (ISS) $>15$ presenting within 6 hours of initial injury.

2. Age limit: 14 - 60 years

Exclusion Criteria:

History was elicited from the patient or the para medics / care givers / relatives accompanying the patient to the Emergency Department and following patients were excluded.

- Patients who received more than 2 liters of crystalloids before admission.

- Patients who received blood transfusion before admission.

- Patients with a known bleeding diathesis.

- Patients on anticoagulant medications as elicited from history.

- Patients with a history of liver disease or renal failure.

\section{DATA COLLECTION PROCEDURE:}

After approval from CPSP and Institutional review board (d.u.h.s) 80 patients full filling the inclusion and exclusion criteria were enrolled from Emergency Department and Surgical Unit 4, Civil Hospital Karachi. Written informed consent was taken from care taker. Demographic data including age, gender, time from injury to arrival in the emergency department, mechanism of injury, fluid administration at scene; and on anatomic injury characteristics and scored using Injury severity scoring (ISS) calculated from these data. On the arrival of the patient in the emergency department, the first task of one member of the trauma team was to take a blood sample (arterial or venous) via a direct puncture, with a non-heparinized syringe. The sample was placed in a citrated tube and sent immediately to the hospital's central laboratory. Prothrombin time (PT), Activated partial thromboplastin time (APTT), and International normalized ratio (INR) was determined from this sample, and represented the coagulation state before 
further resuscitation. The coagulation data was collected from the hospital's laboratory computer database. The presence of a coagulopathy was defined as an INR over 1.2 and/or an APTT over 45 seconds (1.5 times the upper limit of institute). Mortality was used as the outcome measure. Patients that were admitted to the surgical unit or intensive care unit following resuscitation and management of injuries were followed for in-hospital mortality on 0,2 nd, 6th day and finally assessed on 14th day. Patients that were discharged within 2 weeks of in hospital admission or the patients that were not admitted in hospital and discharged from the emergency department were considered as survivors.

\section{Results}

In our study 80 patients with blunt and penetrating trauma injury were enrolled. The injury was accidental as well as non-accidental. The in-hospital mortality was compared in patients with coagulopathy and patients without coagulopathy.

There were 43 males and 37 female patients [Table 1].

Accidental injury was present in $46.3 \%$ and $53.8 \%$ had non accidental injury [Table 2].

Blunt trauma was found in 32 patients and penetrating trauma was found in 48 patients [Table 3].

Trauma coagulopathy was present in $12.5 \%$ patients [Table 4].

In hospital mortality was found in $16.3 \%$ patients [Table 5$]$.

The mean age of patients was $27.28 \pm 12.18$ [Table 6].

The amount of fluid given at presentation was $1200 \pm 80 \mathrm{ml}$ [Table 7].

The mean injury severity score was $50.2 \pm 11.18$ [Table 8].

Prothombin time was $13.3 \pm 1.8 \mathrm{sec}$, APTT was $32.3 \pm 2.1$ and mean INR was $1.2 \pm 0.1$ [Table 9].

The inhospital mortality was compared in patients with coagulopathy and patients without coagulopathy and $p$ value was significant 0.00 [Table 10].

Data stratification was significant for age p value 0.016 [Table 11].

Data stratification for gender, mode of injury, mechanism of injury, amount of fluid given at presentation and injury severity score was not significant [Table 12,13,14, 15 and 16].

Table 1: Gender distribution 


\begin{tabular}{|llll|}
\hline \multirow{2}{*}{ Valid } & & Frequency & Percent \\
\cline { 2 - 4 } & Male & 43 & 53.8 \\
\cline { 2 - 4 } & Female & 37 & 46.3 \\
\hline Total & 80 & 100.0 \\
\hline
\end{tabular}

Table 2: Mode of injury

\begin{tabular}{|llll|}
\hline \multirow{3}{*}{ Valid } & & Frequency & Percent \\
\cline { 2 - 4 } & Accidental & 37 & 46.3 \\
\cline { 2 - 4 } & Non-accidental & 43 & 53.8 \\
\cline { 2 - 4 } & Total & 80 & 100.0 \\
\hline
\end{tabular}

Table 3: Mechanism of injury

\begin{tabular}{|clll|}
\hline \multirow{3}{*}{ Valid } & & Frequency & Percent \\
\cline { 2 - 4 } & Blunt & 32 & 40.0 \\
\cline { 2 - 4 } & Penetrating & 48 & 60.0 \\
\cline { 2 - 4 } & Total & 80 & 100.0 \\
\hline
\end{tabular}

Table 4: Frequency of trauma coagulopathy

\begin{tabular}{|clll|}
\hline \multirow{4}{*}{ Valid } & & Frequency & Percent \\
\cline { 2 - 4 } & Yes & 10 & 12.5 \\
\cline { 2 - 4 } & No & 70 & 87.5 \\
\cline { 2 - 3 } & Total & 80 & 100.0 \\
\hline
\end{tabular}

Table 5: Frequency of in hospital mortality 


\begin{tabular}{|clll|}
\hline \multirow{3}{*}{ Valid } & & Frequency & Percent \\
\cline { 2 - 4 } & Discharge & 67 & 83.8 \\
\cline { 2 - 4 } & Expire & 13 & 16.3 \\
\cline { 2 - 4 } & Total & 80 & 100.0 \\
\hline
\end{tabular}

Table 6: Age distribution of sampled population

\begin{tabular}{|llllll|}
\hline \multicolumn{6}{|c|}{ Descriptive Statistics age } \\
& $\mathrm{N}$ & Minimum & Maximum & Mean & Std. Deviation \\
\hline Age & 80 & 15 & 60 & 27.28 & 12.181 \\
\hline
\end{tabular}

Table 7: Amount of fluid given

\begin{tabular}{|c|c|c|c|c|c|}
\hline \multicolumn{6}{|c|}{ Descriptive Statistics amount of fluid } \\
\hline & $\mathrm{N}$ & Minimum & Maximum & Mean & Std. Deviation \\
\hline Age & 80 & $500 \mathrm{ml}$ & $1500 \mathrm{ml}$ & $1200 \mathrm{ml}$ & $80.18 \mathrm{ml}$ \\
\hline
\end{tabular}

Table 8: Injury severity score

\begin{tabular}{|llllll|}
\hline \multicolumn{6}{|c|}{ Descriptive Statistics injury severity score } \\
& N & Minimum & Maximum & Mean & Std. Deviation \\
Age & 80 & 16 & 74 & 50.28 & 11.181 \\
\hline
\end{tabular}

Table 9: PT, APTT, INR 


\begin{tabular}{|llllll|}
\hline \multicolumn{7}{|c|}{ Descriptive Statistics PT, APTT, INR } \\
& $\mathrm{N}$ & Minimum & Maximum & Mean & Std. Deviation \\
\hline PT & 80 & 11 & 21 & 13.3 & 1.81 \\
\hline APTT & 80 & 26 & 40 & 32.3 & 2.1 \\
\hline INR & 80 & 1 & 1.6 & 1.2 & 0.1 \\
\hline
\end{tabular}

Table 10: Comparison of in hospital mortality in patients with coagulopathy and without coagulopathy

\begin{tabular}{|c|c|c|c|c|c|}
\hline \multicolumn{6}{|c|}{ Cross tabulation inhospital mortality and Trauma coagulopathy } \\
\hline & & & \multicolumn{2}{|c|}{ Trauma coagulopathy } & \multirow[t]{2}{*}{ Total } \\
\hline & & & Yes & No & \\
\hline \multirow[t]{4}{*}{ Inhospital mortality } & Discharge & Count & 3 & 64 & 67 \\
\hline & & $\%$ within inhospital mortality & $4.5 \%$ & $95.5 \%$ & $100.0 \%$ \\
\hline & Expire & Count & 7 & 6 & 13 \\
\hline & & $\%$ within inhospital mortality & $53.8 \%$ & $46.2 \%$ & $100.0 \%$ \\
\hline \multirow{2}{*}{\multicolumn{2}{|c|}{ Total }} & Count & 10 & 70 & 80 \\
\hline & & $\%$ within inhospital mortality & $12.5 \%$ & $87.5 \%$ & $100.0 \%$ \\
\hline
\end{tabular}

Table 11: Data stratification for age 


\begin{tabular}{|c|c|c|c|c|c|c|}
\hline \multicolumn{7}{|c|}{ Cross tabulation Age, inhospital mortality and Trauma coagulopathy } \\
\hline \multicolumn{4}{|c|}{ Trauma coagulopathy } & \multicolumn{2}{|c|}{ Inhospital mortality } & \multirow[t]{2}{*}{ Total } \\
\hline & & & & Discharge & Expire & \\
\hline \multirow[t]{6}{*}{ Yes } & \multirow[t]{4}{*}{ Age } & \multirow[t]{2}{*}{$13-40$ years } & Count & 1 & 7 & 8 \\
\hline & & & $\%$ within Age & $12.5 \%$ & $87.5 \%$ & $100.0 \%$ \\
\hline & & \multirow[t]{2}{*}{$41-65$ years } & Count & 2 & 0 & 2 \\
\hline & & & $\%$ within Age & $100.0 \%$ & $0.0 \%$ & $100.0 \%$ \\
\hline & \multirow{2}{*}{\multicolumn{2}{|c|}{ Total }} & Count & 3 & 7 & 10 \\
\hline & & & $\%$ within Age & $30.0 \%$ & $70.0 \%$ & $100.0 \%$ \\
\hline \multirow[t]{6}{*}{ No } & \multirow[t]{4}{*}{ Age } & \multirow[t]{2}{*}{$13-40$ years } & Count & 49 & 6 & 55 \\
\hline & & & $\%$ within Age & $89.1 \%$ & $10.9 \%$ & $100.0 \%$ \\
\hline & & \multirow[t]{2}{*}{$41-65$ years } & Count & 15 & 0 & 15 \\
\hline & & & $\%$ within Age & $100.0 \%$ & $0.0 \%$ & $100.0 \%$ \\
\hline & \multirow{2}{*}{\multicolumn{2}{|c|}{ Total }} & Count & 64 & 6 & 70 \\
\hline & & & $\%$ within Age & $91.4 \%$ & $8.6 \%$ & $100.0 \%$ \\
\hline \multirow[t]{6}{*}{ Total } & \multirow[t]{4}{*}{ Age } & \multirow[t]{2}{*}{$13-40$ years } & Count & 50 & 13 & 63 \\
\hline & & & $\%$ within Age & $79.4 \%$ & $20.6 \%$ & $100.0 \%$ \\
\hline & & \multirow[t]{2}{*}{$41-65$ years } & Count & 17 & 0 & 17 \\
\hline & & & $\%$ within Age & $100.0 \%$ & $0.0 \%$ & $100.0 \%$ \\
\hline & \multirow{2}{*}{\multicolumn{2}{|c|}{ Total }} & Count & 67 & 13 & 80 \\
\hline & & & $\%$ within Age & $83.8 \%$ & $16.2 \%$ & $100.0 \%$ \\
\hline \multicolumn{7}{|c|}{$p$-value $=0.016$ significant } \\
\hline
\end{tabular}

Table 12: Data stratification for gender 


\begin{tabular}{|c|c|c|c|c|c|c|}
\hline \multicolumn{7}{|c|}{ Cross tabulation Gender, inhospital mortality and Trauma coagulopathy } \\
\hline \multicolumn{4}{|c|}{ Trauma coagulopathy } & \multicolumn{2}{|c|}{ Inhospital mortality } & \multirow[t]{2}{*}{ Total } \\
\hline & & & & Discharge & Expire & \\
\hline \multirow[t]{6}{*}{ Yes } & \multirow[t]{4}{*}{ Gender } & \multirow[t]{2}{*}{ Male } & Count & 2 & 2 & 4 \\
\hline & & & $\%$ within Gender & $50.0 \%$ & $50.0 \%$ & $100.0 \%$ \\
\hline & & \multirow[t]{2}{*}{ Female } & Count & 1 & 5 & 6 \\
\hline & & & $\%$ within Gender & $16.7 \%$ & $83.3 \%$ & $100.0 \%$ \\
\hline & \multirow{2}{*}{\multicolumn{2}{|c|}{ Total }} & Count & 3 & 7 & 10 \\
\hline & & & $\%$ within Gender & $30.0 \%$ & $70.0 \%$ & $100.0 \%$ \\
\hline \multirow[t]{6}{*}{ No } & \multirow[t]{4}{*}{ Gender } & \multirow[t]{2}{*}{ Male } & Count & 35 & 4 & 39 \\
\hline & & & $\%$ within Gender & $89.7 \%$ & $10.3 \%$ & $100.0 \%$ \\
\hline & & \multirow[t]{2}{*}{ Female } & Count & 29 & 2 & 31 \\
\hline & & & $\%$ within Gender & $93.5 \%$ & $6.5 \%$ & $100.0 \%$ \\
\hline & \multirow{2}{*}{\multicolumn{2}{|c|}{ Total }} & Count & 64 & 6 & 70 \\
\hline & & & $\%$ within Gender & $91.4 \%$ & $8.6 \%$ & $100.0 \%$ \\
\hline \multirow[t]{6}{*}{ Total } & \multirow[t]{4}{*}{ Gender } & \multirow[t]{2}{*}{ Male } & Count & 37 & 6 & 43 \\
\hline & & & $\%$ within Gender & $86.0 \%$ & $14.0 \%$ & $100.0 \%$ \\
\hline & & \multirow[t]{2}{*}{ Female } & Count & 30 & 7 & 37 \\
\hline & & & $\%$ within Gender & $81.1 \%$ & $18.9 \%$ & $100.0 \%$ \\
\hline & \multirow{2}{*}{\multicolumn{2}{|c|}{ Total }} & Count & 67 & 13 & 80 \\
\hline & & & $\%$ within Gender & $83.8 \%$ & $16.2 \%$ & $100.0 \%$ \\
\hline
\end{tabular}

Table 13: Data stratification for mode of injury 


\begin{tabular}{|c|c|c|c|c|c|c|}
\hline Cross & abulation I & of Injury, inh & al mortality a & d Trauma co & & \\
\hline Traum & coagulop & & & Inhospital & Total & \\
\hline & & & & Discharge & Expire & \\
\hline Yes & Mode of & Accidental & Count & 0 & 2 & 2 \\
\hline & & & Percentage & $0.0 \%$ & $100.0 \%$ & $100.0 \%$ \\
\hline & & Non- & Count & 3 & 5 & 8 \\
\hline & & & Percentage & $37.5 \%$ & $62.5 \%$ & $100.0 \%$ \\
\hline & Total & & Count & 3 & 7 & 10 \\
\hline & & & Percentage & $30.0 \%$ & $70.0 \%$ & $100.0 \%$ \\
\hline No & Mode of & Accidental & Count & 32 & 3 & 35 \\
\hline & & & Percentage & $91.4 \%$ & $8.6 \%$ & $100.0 \%$ \\
\hline & & Non- & Count & 32 & 3 & 35 \\
\hline & & & Percentage & $91.4 \%$ & $8.6 \%$ & $100.0 \%$ \\
\hline & Total & & Count & 64 & 6 & 70 \\
\hline & & & Percentage & $91.4 \%$ & $8.6 \%$ & $100.0 \%$ \\
\hline Total & Mode of & Accidental & Count & 32 & 5 & 37 \\
\hline & & & Percentage & $86.5 \%$ & $13.5 \%$ & $100.0 \%$ \\
\hline & & Non- & Count & 35 & 8 & 43 \\
\hline & & & Percentage & $81.4 \%$ & $18.6 \%$ & $100.0 \%$ \\
\hline & Total & & Count & 67 & 13 & 80 \\
\hline & & & Percentage & $83.8 \%$ & $16.2 \%$ & $100.0 \%$ \\
\hline
\end{tabular}

Table 14: Data stratification for mechanism of injury 


\begin{tabular}{|c|c|c|c|c|c|c|}
\hline \multicolumn{7}{|c|}{ Cross tabulation Mechanism of Injury, inhospital mortality and Trauma coagulopathy } \\
\hline \multicolumn{4}{|c|}{ Trauma coagulopathy } & \multicolumn{2}{|c|}{ Inhospital mortality } & \multirow[t]{2}{*}{ Total } \\
\hline & & & & Discharge & Expire & \\
\hline \multirow[t]{6}{*}{ Yes } & \multirow[t]{4}{*}{ Mechanism of Injury } & \multirow[t]{2}{*}{ Blunt } & Count & 2 & 4 & 6 \\
\hline & & & Percentage & $33.3 \%$ & $66.7 \%$ & $100.0 \%$ \\
\hline & & \multirow[t]{2}{*}{ Penetrating } & Count & 1 & 3 & 4 \\
\hline & & & Percentage & $25.0 \%$ & $75.0 \%$ & $100.0 \%$ \\
\hline & \multirow[t]{2}{*}{ Total } & & Count & 3 & 7 & 10 \\
\hline & & & Percentage & $30.0 \%$ & $70.0 \%$ & $100.0 \%$ \\
\hline \multirow[t]{6}{*}{ No } & \multirow[t]{4}{*}{ Mechanism of Injury } & \multirow[t]{2}{*}{ Blunt } & Count & 23 & 3 & 26 \\
\hline & & & Percentage & $88.5 \%$ & $11.5 \%$ & $100.0 \%$ \\
\hline & & \multirow[t]{2}{*}{ Penetrating } & Count & 41 & 3 & 44 \\
\hline & & & Percentage & $93.2 \%$ & $6.8 \%$ & $100.0 \%$ \\
\hline & \multirow[t]{2}{*}{ Total } & & Count & 64 & 6 & 70 \\
\hline & & & Percentage & $91.4 \%$ & $8.6 \%$ & $100.0 \%$ \\
\hline \multirow[t]{6}{*}{ Total } & \multirow[t]{4}{*}{ Mechanism of Injury } & \multirow[t]{2}{*}{ Blunt } & Count & 25 & 7 & 32 \\
\hline & & & Percentage & $78.1 \%$ & $21.9 \%$ & $100.0 \%$ \\
\hline & & \multirow[t]{2}{*}{ Penetrating } & Count & 42 & 6 & 48 \\
\hline & & & Percentage & $87.5 \%$ & $12.5 \%$ & $100.0 \%$ \\
\hline & \multirow[t]{2}{*}{ Total } & & Count & 67 & 13 & 80 \\
\hline & & & Percentage & $83.8 \%$ & $16.2 \%$ & $100.0 \%$ \\
\hline
\end{tabular}

Table 15: Data stratification for amount of fluid 


\begin{tabular}{|c|c|c|c|c|c|c|}
\hline \multicolumn{7}{|c|}{ Cross tabulation Amount of fluid, inhospital mortality and Trauma coagulopathy } \\
\hline \multicolumn{4}{|c|}{ Trauma coagulopathy } & \multicolumn{2}{|c|}{ Inhospital mortality } & \multirow[t]{2}{*}{ Total } \\
\hline & & & & Discharge & Expire & \\
\hline \multirow[t]{6}{*}{ Yes } & \multirow[t]{4}{*}{ Amount of fluid } & \multirow[t]{2}{*}{ Less than $1000 \mathrm{ml}$} & Count & 1 & 2 & 3 \\
\hline & & & Percentage & $33.3 \%$ & $66.7 \%$ & $100.0 \%$ \\
\hline & & \multirow[t]{2}{*}{ More than $1000 \mathrm{ml}$} & Count & 2 & 5 & 7 \\
\hline & & & Percentage & $28.6 \%$ & $71.4 \%$ & $100.0 \%$ \\
\hline & \multirow[t]{2}{*}{ Total } & & Count & 3 & 7 & 10 \\
\hline & & & Percentage & $30.0 \%$ & $70.0 \%$ & $100.0 \%$ \\
\hline \multirow[t]{6}{*}{ No } & \multirow[t]{4}{*}{ Amount of fluid } & \multirow[t]{2}{*}{ Less than $1000 \mathrm{ml}$} & Count & 42 & 3 & 45 \\
\hline & & & Percentage & $93.3 \%$ & $6.7 \%$ & $100.0 \%$ \\
\hline & & \multirow[t]{2}{*}{ More than $1000 \mathrm{ml}$} & Count & 22 & 3 & 25 \\
\hline & & & Percentage & $88.0 \%$ & $12.0 \%$ & $100.0 \%$ \\
\hline & \multirow[t]{2}{*}{ Total } & & Count & 64 & 6 & 70 \\
\hline & & & Percentage & $91.4 \%$ & $8.6 \%$ & $100.0 \%$ \\
\hline \multirow[t]{6}{*}{ Total } & \multirow[t]{4}{*}{ Amount of fluid } & \multirow[t]{2}{*}{ Less than $1000 \mathrm{ml}$} & Count & 43 & 5 & 48 \\
\hline & & & Percentage & $89.6 \%$ & $10.4 \%$ & $100.0 \%$ \\
\hline & & \multirow[t]{2}{*}{ More than $1000 \mathrm{ml}$} & Count & 24 & 8 & 32 \\
\hline & & & Percentage & $75.0 \%$ & $25.0 \%$ & $100.0 \%$ \\
\hline & \multirow{2}{*}{\multicolumn{2}{|c|}{ Total }} & Count & 67 & 13 & 80 \\
\hline & & & Percentage & $83.8 \%$ & $16.2 \%$ & $100.0 \%$ \\
\hline
\end{tabular}

Table 16: Data stratification for injury severity score 


\begin{tabular}{|c|c|c|c|c|c|c|}
\hline \multicolumn{7}{|c|}{ Cross tabulation Injury severity score, inhospital mortality and Trauma coagulopathy } \\
\hline \multicolumn{4}{|c|}{ Trauma coagulopathy } & \multicolumn{2}{|c|}{ Inhospital mortality } & \multirow[t]{2}{*}{ Total } \\
\hline & & & & Discharge & Expire & \\
\hline \multirow[t]{8}{*}{ Yes } & \multirow[t]{6}{*}{ Injury severity score } & \multirow[t]{2}{*}{ serious $16-24$} & Count & 2 & 2 & 4 \\
\hline & & & Percentage & $50.0 \%$ & $50.0 \%$ & $100.0 \%$ \\
\hline & & \multirow[t]{2}{*}{ severe $25-49$} & Count & 1 & 4 & 5 \\
\hline & & & Percentage & $20.0 \%$ & $80.0 \%$ & $100.0 \%$ \\
\hline & & \multirow[t]{2}{*}{ critical 50-74 } & Count & 0 & 1 & 1 \\
\hline & & & Percentage & $0.0 \%$ & $100.0 \%$ & $100.0 \%$ \\
\hline & \multirow[t]{2}{*}{ Total } & & Count & 3 & 7 & 10 \\
\hline & & & Percentage & $30.0 \%$ & $70.0 \%$ & $100.0 \%$ \\
\hline \multirow[t]{8}{*}{ No } & \multirow[t]{6}{*}{ Injury severity score } & \multirow[t]{2}{*}{ serious $16-24$} & Count & 28 & 0 & 28 \\
\hline & & & Percentage & $100.0 \%$ & $0.0 \%$ & $100.0 \%$ \\
\hline & & \multirow[t]{2}{*}{ severe $25-49$} & Count & 23 & 3 & 26 \\
\hline & & & Percentage & $88.5 \%$ & $11.5 \%$ & $100.0 \%$ \\
\hline & & \multirow[t]{2}{*}{ critical 50-74 } & Count & 13 & 3 & 16 \\
\hline & & & Percentage & $81.2 \%$ & $18.8 \%$ & $100.0 \%$ \\
\hline & \multirow[t]{2}{*}{ Total } & & Count & 64 & 6 & 70 \\
\hline & & & Percentage & $91.4 \%$ & $8.6 \%$ & $100.0 \%$ \\
\hline \multirow[t]{8}{*}{ Total } & \multirow[t]{6}{*}{ Injury severity score } & \multirow[t]{2}{*}{ serious $16-24$} & Count & 30 & 2 & 32 \\
\hline & & & Percentage & $93.8 \%$ & $6.2 \%$ & $100.0 \%$ \\
\hline & & \multirow[t]{2}{*}{ severe $25-49$} & Count & 24 & 7 & 31 \\
\hline & & & Percentage & $77.4 \%$ & $22.6 \%$ & $100.0 \%$ \\
\hline & & \multirow[t]{2}{*}{ critical 50-74 } & Count & 13 & 4 & 17 \\
\hline & & & Percentage & $76.5 \%$ & $23.5 \%$ & $100.0 \%$ \\
\hline & \multirow[t]{2}{*}{ Total } & & Count & 67 & 13 & 80 \\
\hline & & & Percentage & $83.8 \%$ & $16.2 \%$ & $100.0 \%$ \\
\hline
\end{tabular}




\section{Discussion}

For decades, the art of medically managing the trauma patient was just that, an art. A paucity of scientific information existed until the beginning of Operation Desert Shield in 1990 when the problem of massive trauma, coagulopathy, and transfusion was thrust prominently into the global spotlight. The United States Centers for Disease Control and Prevention in 2014 ranked unintentional injury as the leading cause of death among ages 1-44 [12].Trauma coagulopathy is an early, primary post-injury coagulopathy. It is associated with an increase in mortality, even when controlling for crystalloids, vital signs, injury severity and head injury. In our study the mean age of patients was $27.28 \pm 12.18$, there were 43 males and 37 female patients. Accidental injury was present in $46.3 \%$ and $53.8 \%$ had non accidental injury. Blunt trauma was found in 32 patients and penetrating trauma was found in 48 patients. Trauma coagulopathy was present in $12.5 \%$ patients. In hospital mortality was found in $16.3 \%$ patients. The mean injury severity score was $50.2 \pm 11.18$. The inhospital mortality was compared in patients with coagulopathy and patients without coagulopathy and $p$ value was significant 0.00 . Data stratification was significant for age $p$ value 0.016 . Data stratification for gender, mode of injury, mechanism of injury, amount of fluid given at presentation and injury severity score was not significant.

Our results were comparable to other international studies. A high prevalence of ATC has been reported in Uganda with 54\% [13], while an Australian study found that only $9.0 \%$ of their traumatic patients met the definition of traumatic coagulopathy [14]. Two studies from Europe have reported the prevalence of ATC in the range of $24 \%$ to $34 \%[15,16]$.

Mitra stated that Acute traumatic coagulopathy is observed in $10-25 \%$ of patients post major trauma and its management forms an integral part of haemostatic resuscitation There were 772 patients included in this study with a median ISS of 29 (19-41), with $91.7 \%$ blunt trauma. All-cause in-hospital mortality was $17.5 \%$, while 77 (9.7\%) patients died early. Increasing age (OR 1.04), a GCS of 3-8 (OR 5.05), and the presence of acute coagulopathy (OR 8.77) were significant independent variables associated with early death [17].

A study by MacLeoab et al [18] on 701 patients gave similar results. There was $75.3 \%$ male, $25.7 \%$ penetrating, with a mean age of 39 years. The overall mortality was $7.3 \%$. ATC occurred in 114 patients (16.3\%) and was found to be independently associated with death (odds of death (per $0.10 \mathrm{~s}$ increase in PT): $1.10, p=0.001$ ) However, even mildly injured patients, who had an ISS $<16$, normal RTS score, and no fluid resuscitation, had an ETIC prevalence of $11.7 \%$ (11/94).

Hojjat [19] conducted a study on 246 patients with the mean age of $36.57 \pm 17.11$ years were included (88.2\% male). The mean injury severity score (ISS) was $21.83 \pm 7.37(16-54)$. Patients were resuscitated with $676.83 \pm 452.02(0-1500) \mathrm{ml}$ intravenous fluid before arriving at the ED. The maximum and minimum frequencies of ATC were $31.3 \%$ based on PTT $>36 \mathrm{~s}$ and $2.4 \%$ based on PT $>18 \mathrm{~s}$, respectively. There was a significant association between the occurrence of ATC (PT ratio $>1.2)$ and ISS $>23(p=$ $0.001)$, abdominal abbreviated injury score (AIS) $>3(p=0.003)$, base deficit $>4(p=0.019)$, pulse rate $>$ $90 /$ minute $(p=0.041)$, and $\mathrm{pH}<7.30(\mathrm{p}=0.043)$. 


\section{Conclusion}

Acute traumatic coagulopathy (ATC) occurs after severe injury and shock and is associated with increased bleeding, morbidity, and mortality. Our study concludes that a significant proportion of major trauma patients were coagulopathic. Initial coagulation profile is useful in predicting outcomes for major trauma patients. A high proportion of patients arrive in the emergency department with an established clotting disorder, we suggest that a coagulation profile is an important laboratory investigation that should be performed early in the workup of trauma patients even in resource limited environments in order to provide directed and aggressive management to the patients at high risk and to reduce morbidity and improve survival.

\section{Declarations}

FUNDING: Not applicable

CONFLICTS OF INTEREST: Author has no conflicts of interest to declare.

ETHIC APPROVAL: Study was conducted after taking approval from College of Physicians and Surgeons Pakistan (CPSP) and Institutional Review Board of Dow University of Health Sciences (D.U.H.S).

CONSENT TO PARTICIPATE: Written and informed consent was taken from the care taker of the patients.

AVAILABILITY OF DATA AND MATERIAL: Data available within the article.

CODE AVAILABILITY: Not applicable

\section{AUTHORS' CONTRIBUTIONS:}

Conceptualization: Fariha Ashraf

Methodology: Fariha Ashraf, M. Faisal Ibrahim

Formal analysis and investigation: Rabia Feroz, Abdul Wasey

Writing - original draft preparation: Fariha Ashraf, M. Arsalan

Writing - review and editing: Rabia Feroz, M. Faisal Ibrahim, M. Arsalan

Resources: Fariha Ashraf, Abdul Wasey

Supervision: M. Saeed Quraishy

\section{References}


1. Mathers CD, Loncar D. Projections of global mortality and burden of disease from 2002 to 2030. PLoS Med. 2006;3(11):2442

2. Mujuni E, Wangoda R, Ongom P, Galukande M. Acute traumatic coagulopathy among major trauma patients in an urban tertiary hospital in sub Saharan Africa. BMC Emerg Med.2012;12(1):16.

3. Kauvar DS, Lefering R, Wade CE. Impact of hemorrhage on trauma outcome: an overview of epidemiology, clinical presentations, and therapeutic considerations. J Trauma Acute Care Surg. 2006;60(6):S3-S11.

4. Frith D, Goslings J, Gaarder C, Maegele M, Cohen M, Allard S, et al. Definition and drivers of acute traumatic coagulopathy: clinical and experimental investigations. J. Thromb. Haemost.2010;8(9):1919-25.

5. Nardi G, Agostini V, Rondinelli B, Russo E, Bastianini B, Bini G, et al. Trauma-induced coagulopathy: impact of the early coagulation support protocol on blood product consumption, mortality and costs. Critic Care. 2015;19(1):83.

6. Davenport R. Pathogenesis of acute traumatic coagulopathy. Transfusion. 2013;53(S1).

7. Simmons J, Powell M. Acute traumatic coagulopathy: pathophysiology and resuscitation. $\mathrm{Br} \mathrm{J}$ Anaesth. 2016;117(suppl 3):11131-1143.

8. Acute and delayed coagulopathy are related to outcome in patients with isolated traumatic brain injury. Critic Care. 2011;15(1):R2,

9. Brohi K, Cohen MJ, Ganter MT, Matthay MA, Mackersie RC, Pittet J-F. Acute traumatic coagulopathy: initiated by hypoperfusion: modulated through the protein C pathway? Ann. Surg. 2007;245(5):812.

10. Cap A, Hunt B. Acute traumatic coagulopathy. Curr Opin Crit Care. 2014;20(6):638-45.

11. Cap A, Hunt B. Acute traumatic coagulopathy. Curr Opin Crit Care. 2014;20(6):638-45.

12. 10 Leading Causes of Death By Age Group, UnitedStates-2014. 2014. Available from http://www.cdc.gov/injury/wisqars/leadingcauses.html (accessed 23 May 2016

13. Mujuni E, Wangoda R, Ongom P, Galukande M. Acute traumatic coagulopathy among major trauma patients in an urban tertiary hospital in sub Saharan Africa. BMC Emerg Med. 2012;12:16.

14. Mitra B, Cameron PA, Mori A, Maini A, Fitzgerald M, Paul E, et al. Early prediction of acute traumatic coagulopathy. Resuscitation. 2011;82(9):1208-13.

15. Brohi K, Singh J, Heron M, Coats T. Acute traumatic coagulopathy. The Journal of trauma. 2003;54(6):1127-30.

16. Maegele M, Paffrath T, Bouillon B. Acute traumatic coagulopathy in severe injury: incidence, risk stratification, and treatment options. Deutsches Ärzteblatt international. 2011;108:827-35.

17. Mitra, Cameron PA, Mori A, Fitzgerald M. Acute coagulopathy and early deaths post major trauma. Injury. 2012 Jan;43(1):22-5.

18. MacLeod J, Winkler A, McCoy C, Hillyer C, Shaz B. Early trauma induced coagulopathy (ETIC): Prevalence across the injury spectrum. Injury. 2014;45(5):910-915. 
19. Derakhshanfar H, Vafaei A, Tabatabaey A, Noori S. Prevalence and Associated Factors of Acute Traumatic Coagulopathy; a Cross Sectional Study. Emerg (Tehran). 2017; 5(1): e58. 